\title{
An ethical framework for public health immunisation programs
}

\author{
David Isaacs \\ Department of Infectious Diseases, Children's Hospital \\ at Westmead \\ Email:david.isaacs@health.nsw.gov.au
}

\begin{abstract}
This paper presents seven ethical principles associated with the implementation of immunisation programs. For a public health immunisation program to be ethically justifiable, its principles and operation should be based on sound ethical values: the program should benefit the individual and the community; targeted diseases should be sufficiently severe and frequent to justify the risks and expense of the program, and vulnerable groups within the population should be targeted. The principles also deal with the obligation to monitor for adverse events and for disease incidence to ensure safety and effectiveness. When immunisations are voluntary, vaccine recipients or their parents or carers should be given sufficient information to make autonomous, informed decisions and incentives to participate in public health immunisation programs should not be coercive. Public health immunisation programs depend on mutual trust, which may be threatened by circumstances such as excessive media publicity about adverse events associated with vaccines.
\end{abstract}

Immunisation is one of the most successful of all public health interventions, responsible for the eradication of smallpox, the near eradication of poliomyelitis and huge reductions in the incidence of many other lethal infectious diseases including diphtheria, measles, Haemophilus influenzae type b (Hib), meningococcal, pneumococcal and rotavirus infections, saving many millions of lives annually. ${ }^{1,2}$ Funded immunisation programs reduce inequity, because the socio-economically disadvantaged are at greater risk from many infections. ${ }^{2}$ If immunisation levels fall, diseases may return, exemplified by the major diphtheria outbreak in Russia in the 1990s with over 140000 cases and over 4000 deaths. ${ }^{3}$ Immunisation also prevents some infection-related cancers, notably liver cancer through hepatitis B virus vaccine ${ }^{4}$ and cervical cancer through human papillomavirus (HPV) vaccine. ${ }^{5}$

Immunisation programs cause ethical challenges, because they often involve mass immunisation of individuals to benefit not only the individual, but also the population, and because the individuals are often children who are too young to make their own choices. The risks and benefits for an individual vary depending on factors such as age, disease incidence and immunisation levels, raising concerns of autonomy, liberty and justice that may conflict. For example, parents may want to exercise their autonomy not to immunise their school-aged child, but other parents may argue that this decision puts their child at unjustifiable risk. On the whole, however, the majority of the world population supports immunisation programs.

New vaccines bring new ethical challenges. The high cost of vaccine development is often reflected in high prices, making it difficult to demonstrate cost effectiveness. The new zoster vaccine, which has been assessed as cost effective by the Australian Pharmaceutical Benefits Advisory Committee, will benefit elderly people, but at a very high fiscal cost, borne by the community. Vaccines which target sexually transmitted diseases, for example, HPV vaccine, raise unique ethical challenges. The diseases might be prevented by alterations in behaviour (condoms, fidelity or abstinence) but the HPV vaccine is usually administered before sexual debut, at an age when the child may or may not be competent to give individual consent. In the USA, the introduction of HPV vaccine was associated with a moral backlash from conservatives who argued, obscurely, that it would increase promiscuity. The success of immunisation programs depends on public trust, which can be damaged if surveillance mechanisms are not in place to monitor vaccine-adverse events and to deal with safety concerns promptly.

For a public health immunisation program to be ethically justifiable, its principles and operation should be based on sound ethical values. Verweij and Dawson outlined seven ethical principles for collective immunisation programs. ${ }^{6}$ This paper develops this concept further, to outline the ethical basis for seven over-arching principles in the light of the emergence of new and future vaccines. 


\section{Ethical principles for public health}

\section{immunisation programs}

Seven ethical principles that can be applied to considering an immunisation program are:

1. Benefits. The program should benefit the individual and the community significantly. The burden of disease should be sufficient, in terms of severity and frequency, to justify the risks and expense of the program.

2. Risks. Program providers should monitor assiduously for adverse events to ensure the program is as safe as possible.

3. Effectiveness. Program providers should monitor that the program is effective and should halt or alter the program if it is or it becomes ineffective.

4. Equity and justice. The program should be cost effective in comparison with competing health-care interventions. Vulnerable, disadvantaged groups within the population should be targeted for special vaccines if possible.

5. Autonomy. Vaccine recipients and the parents or carers of children or adults not competent to make their own decision should be given sufficient information to make autonomous, informed decisions about the risks and benefits of immunisations. Any incentives to participate and any disincentives for failure to participate in public health immunisation programs should not be so excessive that they are effectively coercive.

6. Reciprocity. People who suffer rare, serious complications of public health immunisation programs should receive adequate medical care and there is a strong ethical argument that governments should have no-fault compensation schemes.

7. Trust. Public health immunisation programs depend on mutual trust, which may be threatened by circumstances. Measures to improve public consultation regarding decisions about public health immunisation programs will improve their ethical status.

\section{Benefits}

It is generally accepted that an immunisation program should benefit the individual and the community. Many but not all immunisation programs confer herd immunity, meaning that immunisation of a proportion of the population against an infectious disease protects other members of the population, both unimmunised and immunised, by reducing disease transmission. This herd immunity is unique to immunisation programs, although it could be argued that smoking prevention programs and programs to reduce drink driving also protect others and confer a form of herd immunity.

Some immunisations benefit the individual but provide no herd immunity because infection is not passed from person to person, e.g. tetanus and rabies. Therefore, how is it ethically justifiable to include tetanus immunisation in a community program? The American Academy of Pediatrics has argued that immunisation against infection not only benefits the individual, but can also benefit the public by preventing the societal costs of medical care. ${ }^{7}$

Because public immunisation programs involve large populations, they carry inherent risks and burdens, and so should target diseases that cause high morbidity and mortality (e.g. diphtheria, tetanus, poliomyelitis, measles) or that are so contagious that, although usually mild, the absolute numbers of severe cases is significant (e.g. chickenpox).

The benefits achieved by immunisation should be better than those obtained by alternative options, either personal or community based. For example, smokers are at increased risk of pneumococcal pneumonia. A decision to fund pneumococcal vaccine for smokers might be considered a poor public health response if a program to reduce smoking was likely to be more cost effective.

\section{Risks}

Most vaccines are given to a large population of mainly healthy individuals, so large numbers of individuals may be affected by rare adverse events. As such, there is an obligation for health authorities to ensure surveillance of adverse events and a timely response to any emerging adverse events, particularly with new vaccines. Licensure studies may involve thousands of individuals but still not have the power to detect very rare but very serious adverse events, such as intussusception following rotavirus vaccine in young children, emphasising the importance of postlicensure surveillance for adverse events. The relative contribution that industry and government should make to funding surveillance is debatable and possibly negotiable, but there is an ethical onus on health authorities to ensure the safety of vaccines and indeed of the whole program, including the way vaccines are administered.

At a population level, the benefits of immunisation should outweigh the risks. As immunisation levels rise, however, the disease becomes rare and the risk to any individual child at any given point in time from the vaccine may be greater than the risk of contracting the disease. For example, measles vaccine carries a one in a million risk of causing encephalitis, compared with a risk of about one in a thousand from wild-type measles. ${ }^{1}$ If there is no circulating measles, the risk from the vaccine may exceed the risk that the child will contract measles and develop a complication. However, the individual's risk can change, e.g. if a measles outbreak occurs or if the child travels to an endemic country.

If all parents decided not to immunise their children, epidemics would recur. If just one or two elect not to immunise, they can be seen as 'free riders' on the rest of the population, although an elective decision to free ride is a less common reason for failure to immunise than family chaos or a genuine belief that vaccines are ineffective. ${ }^{8}$ 
Most parents elect to immunise their children to the benefit of the whole community.

\section{Effectiveness}

Newer vaccines are often licensed on the basis of immunogenicity data, as opposed to trial-based efficacy data or community-based effectiveness data. Vaccine effectiveness in public health programs may be greater than expected, as happened with the Haemophilus influenzae type $b$ vaccine program, because of unanticipated herd immunity, or may be compromised by phenomena such as the emergence of serotype replacement, as followed the introduction of pneumococcal conjugate vaccines. ${ }^{9}$

As continuing an ineffective immunisation program would be unethical, ongoing disease surveillance is essential, and ineffective programs should be changed or halted. This requirement places an obligation on health authorities to maintain surveillance, although it may be within the purview of the health authority to make vaccine funding, in whole or part, contingent on the vaccine company's funding the surveillance. In Australia, vaccine companies are allowed to increase the price of a vaccine if they can show their funded vaccine is more cost effective than anticipated.

\section{Equity and justice}

The cost of a public health immunisation program is an opportunity cost. The money might be better spent on another public health program such as reducing smoking or screening for bowel cancer. The principle of just distribution of limited resources places a reasonable ethical obligation on public health authorities to ensure that any community immunisation program is likely to be as cost effective as other competing health interventions. Since 2006, Australia has considered vaccines offered through the Pharmaceutical Benefits Scheme largely, but not exclusively, on cost-effectiveness criteria. ${ }^{10}$ The Pharmaceutical Benefits Advisory Committee considers the estimated cost per quality-adjusted life year (QALY) of drugs and vaccines. For national immunisation programs, the Pharmaceutical Benefits Advisory Committee takes advice from the Government's expert immunisation advisory committee, the Australian Technical Advisory Group on Immunisation (ATAGI) on issues such as vaccine efficacy, predicted herd immunity and program feasibility. The Pharmaceutical Benefits Advisory Committee process keeps vaccine prices down, helping to fulfil the Government's obligation to spend health resources wisely.

The principle of equity makes it desirable to target vulnerable, disadvantaged sectors of the population with a higher disease incidence with selective immunisation programs. For example, the decision to routinely provide hepatitis A immunisation to Aboriginal and Torres Strait Islander children was made before the Pharmaceutical Benefits
Advisory Committee involvement in vaccines, on the basis of the greater disease burden in this group than in nonAboriginal and Torres Strait Islander children and on equity grounds. Nowadays the same decision would still be possible, but it would need to be shown that hepatitis A vaccine was cost effective for Aboriginal and Torres Strait Islander children but not for non-Aboriginal and Torres Strait Islander children. In 2006, the Pharmaceutical Benefits Advisory Committee did recommend rotavirus vaccine for all Australian children, even though Aboriginal and Torres Strait Islander children had a far higher burden of serious disease, because rotavirus vaccine was deemed cost effective for all children.

\section{Autonomy}

Respect for autonomy is one of the most important ethical principles. It is accepted in most Western countries that individuals can make autonomous decisions about their health care and about their children's health care which reflect their needs, wishes and values. ${ }^{8}$ Compulsory immunisation infringes that autonomy, and there is a strong argument that immunisation should be voluntary as long as voluntary immunisation levels remain acceptably high. ${ }^{11}$ Currently in Australia, because immunisation levels are high without compulsion, a voluntary immunisation policy is safe and effective. Australia legally compels the wearing of seat belts but not immunisation even though seat belts occasionally damage their wearers, particularly children. ${ }^{12}$ It could be argued, on the communitarian grounds that individual immunisation often protects others, that there is a stronger case for compelling immunisation than for compulsory wearing of seat-belts. However, the invasive nature of immunisation, in terms of the physical act of introducing foreign substances into the body and the potential severity and frequency of adverse events compared with the rarity of adverse events from seat-belts, arguably justify the Australian approach. In contrast, compulsory immunisation laws in different states in the USA have been upheld on several occasions by the courts as a reasonable exercise of the power of the state, even in the absence of an epidemic. ${ }^{7}$

Would compulsory community immunisation ever be ethically justified? Special circumstances, such as the emergence of a devastating, new vaccine-preventable disease, might justify introducing a special compulsory immunisation program if the disease were sufficiently severe and the vaccine safe and effective. In this situation, however, it is likely that voluntary immunisation would increase and might make compulsion unnecessary. It is also possible that immunisation levels might fall, e.g. because of a failure of trust, and that the altered risk-benefit ratio might alter the ethical justification for compulsion.

In Australia, where immunisation is voluntary, high levels of population coverage have been achieved and maintained 
primarily by delivering free vaccines through the National Immunisation Program and through financial incentives to providers and to parents. The ethical validity of financial incentives would be compromised if the inducements constituted a major part of child welfare payments, and refusing immunisation would disadvantage financially challenged families. Currently, families which register as conscientious objectors still receive benefits.

Similarly, draconian and punitive disincentives for parents who do not immunise their children are coercive and infringe parental autonomy. In some staes of the USA, children are not allowed to start school without being immunised. In Australia, unimmunised children can attend school but are excluded during disease outbreaks. This approach is more ethically justifiable because it protects all children: the unimmunised child from catching infection and other children from catching infection from the unimmunised child.

There are circumstances where the ethical justification for a targeted compulsory immunisation program is stronger than for a whole-of-population program. It has been argued that immunisation of health-care workers is justified if there is a high risk of their transmitting an infection to their vulnerable patients, e.g. influenza, although this infringement of the health-care worker's autonomy is only justified if immunisation cannot be achieved voluntarily. ${ }^{13,14}$

Is it ever ethically justifiable to over-ride parental autonomy with regard to an individual child's immunisations? If the risk from disease is high and imminent, e.g. the refusal of rabies vaccine by the parents of a child bitten by a rabid animal constitutes a child protection issue: the best interests of the child over-ride parental autonomy, and compulsion is justified. ${ }^{11}$ A more controversial situation involves the parents of babies born to mothers with chronic hepatitis B infection who refuse vaccine and/or immunoglobulin for their newborn babies. ${ }^{15,16}$ The baby's risk of contracting chronic hepatitis $B$ infection varies depending on whether or not the mother is hepatitis $\mathrm{B} e$ antigen positive or negative and whether the parents refuse vaccine or immunoglobulin or both (in one case, Jehovah's Witness parents refused immunoglobulin as a blood product). ${ }^{16,17}$ Sound ethics requires sound facts: it is important to know the estimated risk to the baby of contracting hepatitis $\mathrm{B}$ in different situations, as a basis for considering the best interests of the child during possible child-care proceedings. ${ }^{17}$

\section{Reciprocity}

Parents who immunise their children as part of a public health program are protecting not only their own child but also the entire community. When the disease incidence is low and immunisation levels are high, the risk to a child of having a serious vaccine-related adverse event may be higher than the risk of developing a complication of the disease. Parents who continue to immunise their children under these circumstances are exhibiting communitarian altruism which strengthens community values.

Currently in Australia, if a child suffers an extremely rare but serious complication of immunisation, such as measles vaccine encephalitis, the family only receive normal health care. While recognising that public medicine covers some of the costs of care incurred from rare vaccine-associated injury, for many injuries there are emotional costs and considerable unfunded financial costs. There is a strong ethical argument based on reciprocity and justice that Australia should follow the lead of the 19 other countries which have implemented no-fault compensation schemes for vaccine injuries. ${ }^{18} \mathrm{An}$ analogy can be drawn with people who contracted an infection by receiving blood or blood products contaminated with human immunodeficiency virus (HIV) before the blood supply was secured,${ }^{19}$ although a stronger analogy would be if an unpaid volunteer contracted HIV or hepatitis $\mathrm{C}$ from donating blood, since both voluntary blood donors and vaccine recipients are exhibiting altruism.

A society that depends on communitarian values to protect its population against infectious diseases has a moral obligation to compensate people who suffer unintended harms as a result of altruistically immunising themselves or their children commensurate with those communitarian values.

\section{Trust}

The availability of different vaccines for the same disease may raise ethical problems which challenge conventional cost-effectiveness considerations of the value of vaccines and introduce other values such as public trust. Live attenuated oral polio vaccines (OPV) are cheaper than killed injected inactivated polio vaccines (IPV). Both are highly effective in eradicating polio in national immunisation programs. OPV is preferred in developing countries, largely because of cost. However, one in every 2.4 million doses of OPV causes vaccine-associated paralytic poliomyelitis, indistinguishable clinically from wild-type polio, ${ }^{20}$ whereas IPV never causes vaccine-associated paralytic poliomyelitis. Australia's decision to change to IPV antedated the Pharmaceutical Benefits Advisory Committee involvement in vaccine decisions. ${ }^{10}$

In the USA, about eight cases of vaccine-associated paralytic poliomyelitis occurred annually from OPV and the change from OPV to IPV was made to avoid such cases occurring and to maintain public trust in the national immunisation program. ${ }^{20}$ In Australia, it was initially estimated to cost over $\$ 100$ million to prevent one case of vaccine-associated paralytic poliomyelitis, an opportunity cost the government could hardly ignore. ${ }^{20}$ However, IPVcontaining combination vaccines became available (consequently, an extra injection was not needed), the price of IPV in the combination vaccines fell and the price of OPV rose. 
At this time, Australia elected to switch from OPV to IPV to prevent any cases of vaccine-associated paralytic poliomyelitis and to maintain public trust in the immunisation program. ${ }^{21}$ The Pharmaceutical Benefits Advisory Committee did not have to compare OPV with IPV, so it is not known whether IPV would have been found to be more or less cost effective than OPV. If it were not, Australia would have been unable to change to IPV under current regulations, which raises the question of whether consideration of public trust should be incorporated into Pharmaceutical Benefits Advisory Committee decision making.

The benefits and harms of public health immunisation are borne by the whole community. There is a strong ethical case for better public consultation about immunisation programs, to include community values into decisionmaking. Lay members on advisory committees may struggle to represent community views. There is increasing interest in direct community involvement through avenues such as telephone surveys, ${ }^{22}$ public meetings, citizens' juries and consensus conferences. ${ }^{8}$

Such consultation can yield important and unexpected information. A telephone survey about HPV vaccination found that $83 \%$ of the public sampled thought HPV vaccination should be given to boys as well as girls, information that is arguably germane to any decision about funding the vaccine for boys in a public health program. ${ }^{22}$

\section{Acknowledgments}

I gratefully acknowledge the insightful and invaluable comments of Associate Professor Ian Kerridge, and the assistance of Bernadette Tobin, Andrea Forde and Helen Marshall.

\section{References}

1. Ada G, Isaacs D. Vaccination, the facts, the fears, the future. Sydney: Allen \& Unwin; 2000.

2. Andre FE, Booy R, Bock HL, Clemens J, Datta SK, John TJ. Vaccination greatly reduces disease, disability, death and inequity worldwide. Bull World Health Organ 2008; 86: 140-6. doi:10.2471/BLT.07.040089

3. Vitek CR, Wharton M. Diphtheria in the former Soviet Union: re-emergence of a pandemic disease. Emerg Infect Dis 1998; 4: 539-50. doi:10.3201/eid0404.980404

4. Chang MH, Chen CJ, Lai MS, Hsu HM, Wu TC, Kong MS et al. Universal hepatitis B vaccination in Taiwan and the incidence of hepatocellular carcinoma in children. Taiwan Childhood Hepatoma Study Group. N Engl J Med 1997; 336: 1855-9. doi:10.1056/NEJM199706263362602

5. Brotherton JML, Fridman M, May CL, Chappell G, Saville AM, Gertig DM. Early effect of the HPV vaccination program on cervical abnormalities in Victoria, Australia: an ecological study. Lancet 2011; 377: 2085-92. doi:10.1016/S0140-6736(11) 60551-5

6. Verweij M, Dawson A. Ethical principles for collective immunisation programs. Vaccine 2004; 22: 3122-6. doi:10.1016/ j.vaccine.2004.01.062
7. Diekema DS. American Academy of Pediatrics Committee on Bioethics. Responding to parental refusals of immunization of children. Pediatrics 2005; 115: 1428-31. doi:10.1542/ peds.2005-0316

8. Leask J, Braunack-Mayer A, Kerridge I. Consent and public engagement in an era of expanded childhood immunisation. J Paediatr Child Health 2011; 47: 603-7. doi:10.1111/ j.1440-1754.2011.02160.x

9. Beall B. Vaccination with the pneumococcal 7-valent conjugate: a successful experiment but the species is adapting. Expert Rev Vaccines 2007; 6: 297-300. doi:10.1586/14760584.6.3.297

10. Australian Government Department of Health and Ageing. Pharmaceutical Benefits Scheme. Available at: http://www. pbs.gov.au/pbs/home (Cited 1 October 2011).

11. Isaacs D, Kilham HA, Marshall H. Should routine childhood immunizations be compulsory? J Paediatr Child Health 2004; 40: 392-6. doi:10.1111/j.1440-1754.2004.00399.x

12. Papavasiliou A, Stanton J, Sinha P, Forder J, Skyrme A. The complexity of seat belt injuries including spinal injury in the pediatric population: a case report of a 6-year-old boy and the literature review. Eur J Emerg Med 2007; 14: 180-3. doi:10.1097/MEJ.0B013e32801430e2

13. Isaacs D, Leask J. Should influenza immunisation be compulsory for health care workers? No. BMJ 2008; 337: a2140. doi:10.1136/bmj.a2140

14. Helms CM, Polgreen PM. Should influenza immunisation be compulsory for health care workers? Yes. BMJ 2008; 337 : a2142. doi:10.1136/bmj.a2142

15. Isaacs D. Children have rights, too. J Paediatr Child Health 2009; 45: 627-8. doi:10.1111/j.1440-1754.2009.01620.x

16. Isaacs D, Kilham HA, Alexander S, Wood N, Buckmaster A, Royle J. Ethical issues in preventing mother-to-child transmission of hepatitis B by immunisation. Vaccine 2011; 29: 6159-62. doi:10.1016/j.vaccine.2011.06.065

17. American Academy of Pediatrics. Religious objections to medical care. Pediatrics 1997; 99: 279-81. doi:10.1542/ peds.99.2.279

18. Kelly HA, Looker C, Isaacs D. A no-fault compensation scheme for serious adverse events attributed to vaccination. Med J Aust 2011; 195: 4-5.

19. Watson CR, Plant AJ. Medical misadventure, indemnity and public health. Aust J Public Health 1993; 17: 88-90. doi:10.1111/j.1753-6405.1993.tb00113.x

20. Centers for Disease Control and Prevention. Poliomyelitis prevention in the United States: introduction of a sequential vaccination schedule of inactivated poliovirus vaccine followed by oral polio-virus vaccine. Recommendations of the Advisory Committee on Immunization Practices (ACIP). [Erratum in: MMWR Morb Mortal Wkly Rep 1997 Feb 28; 46][8]:183 MMWR Recomm Rep 1997; 46: 1-25.

21. Tucker AW, Isaacs D, Burgess M. Cost-effectiveness analysis of changing from live oral polio virus vaccine to inactivated polio virus vaccine in Australia. Aust N Z J Public Health 2001; 25: 411-6.

22. Marshall H, Ryan P, Roberton D, Baghurst P. A cross-sectional survey to assess community attitudes to introduction of human papillomavirus vaccine. Aust N Z J Public Health 2007; 31: 235-42. doi:10.1111/j.1467-842X.2007.00054.x 